\title{
Puzzle and Challenge in Routine Extrahepatic Bile Duct Resection for Advanced Gallbladder Carcinoma
}

\author{
Fei Liu and Fu-Yu Li \\ Department of Biliary Surgery, West China Hospital of Sichuan University, Chengdu, China
}

To the Editor:

Gallbladder carcinoma (GBC) is the most common malignancy of the biliary tract. Curative resection is the only potentially curative treatment for GBC. For advanced GBC, radical resection including gallbladder and liver resection plus regional lymphadenectomy has been widely performed to achieve R0 resection. However, it remains controversial whether routine resection of the extrahepatic bile duct (EHBD) is needed in advanced GBC..$^{1-10}$

Shimizu et al. ${ }^{1}$ analyzed 50 advanced GBC patients with radical resection (including EHBD resection); 30 patients had hepatoduodenal ligament invasion (HDLI), of whom 24 lacked preoperative obstructive jaundice. They found that advanced GBC patients had a higher frequency of HDLI and that invasion was difficult to diagnosis before surgery. Moreover, the 1-, 2-, and 3-year survival rates of the 30 patients with HDLI (50\%, 17\%, and $6 \%$, respectively) were significantly worse than those of the 20 patients without HDLI (75\%, 69\%, and 64\%, respectively). Consequently, they suggested that EHBD resection and lymphadenectomy should be performed in advanced GBC patients. Similar results were reported by Chikamoto et al. ${ }^{2}$ and Kohya and Miyazaki. ${ }^{3}$ In the study conducted by Chikamoto et al., ${ }^{2} 186$ paraffin-embedded specimens from 15 GBC patients with stage T2 disease were analyzed. They demonstrated that there were lymphangions in the submucosal layer of the EHBD and that tumor cells could travel through these channels. Therefore, they recommended routine resection of the EHBD as part of radical resection for T2 GBC. Additionally, Kohya and Miyazaki ${ }^{3}$ reported that T2/T3 GBC patients with EHBD resection had better survival than patients who did not undergo EHBD resection. Thus, they highly recommended the hepatectomy of segments $S 4 b+5$ combined with EHBD resection and lymph node dissection for T2/T3 GBC patients.

However, it had been reported that routine EHBD resection does not prolong survival but rather increases complications. D'Angelica et al. ${ }^{4}$ reported routine EHBD resection had no statistically significant impact on disease-specific survival $(p=0.12)$. Furthermore, EHBD resection was associated with more perioperative complications (19 of 66 patients with EHBD resection and hepaticojejunostomy had abscesses and/or biliary fistulae). Choi et al. ${ }^{5}$ analyzed 71 T2/T3 GBC patients, of whom 31 underwent EHBD resection. There was no difference in the 5-year survival rates between patients without and with EHBD resection (54.2\% vs $34.8 \%, p=0.112$ ), but the length of hospital stay, operative time, proportion of patients needing blood transfusions and proportion of patients developing complications were higher in the group undergoing EHBD resection than in the group not undergoing EHBD resection $(19 \pm 21$ days vs $11 \pm 5$ days, $\mathrm{p}=0.034 ; 345 \pm 136$ minutes vs $186 \pm 93$ minutes, $\mathrm{p}<0.001 ; 12.7 \%$ vs $21.1 \%, p=0.022 ; 4.2 \%$ vs $14.1 \%, p=0.007$, respectively). In the study reported by Gani et al., 449 patients who underwent surgical resection were analyzed. EHBD resection was performed in 109 patients (34.2\%). They found the median number of lymph nodes dissected was similar in patients who did and did not undergo EHBD resection ( 4 vs 3, $\mathrm{p}=0.108$ ). In addition, the median overall survival of patients who underwent EHBD resection was shorter than that of patients who did not undergo EHBD resection (19.2 months vs 32.4 months, $\mathrm{p}<0.001$ ), although EHBD resection did not impact the overall survival rate $(p=0.170)$. However, the proportions of patients with lymph node metastasis and advanced American Joint Committee on Cancer T stage were higher in patients who underwent EHBD resection than in those who did not (52.3\% vs 32.4\%, p<0.001; 57.0\% vs 40.8\%, $\mathrm{p}=0.002$ ). Araida et al. ${ }^{7}$ analyzed $838 \mathrm{R0}$ advanced GBC patients without HDLI or cystic duct invasion. Their results demonstrated that there was no significant differences in the rates of 5-year survival, lymph node metastasis, complications and local recur-

Correspondence to: $\mathrm{Fu}-\mathrm{Yu} \mathrm{Li}$

Department of Biliary Surgery, West China Hospital of Sichuan University, Sichuan Province, Chengdu 610041, China

Tel: +86-2885422465, Fax: +86-2885422465, E-mail: lfy_74@hotmail.com

Received on May 12, 2020. Accepted on May 20, 2020. Published online October 16, 2020.

pISSN 1976-2283 eISSN 2005-1212 https://doi.org/10.5009/gnl20150

@ This is an Open Access article distributed under the terms of the Creative Commons Attribution Non-Commercial License (http://creativecommons.org/licenses/by-nc/4.0) which permits unrestricted non-commercial use, distribution, and reproduction in any medium, provided the original work is properly cited. 
rence along the hepatoduodenal ligament between patients who did and did not undergo EHBD resection. Moreover, Yokomizo et al. ${ }^{8}$ reported that the 5- and 10-year survival rates were lower in patients who underwent EHBD resection than in those who did not (66.7\%, 50.0\% vs $81.1 \%, 66.2 \%$, respectively), although there was no significant difference between the two groups $(\mathrm{p}=0.134)$.

From the above studies, it is unclear whether the routine resection of the EHBD can improve survival in patients with GBC. However, in a study reported recently by our team, ${ }^{9} 213$ advanced GBC patients were analyzed, and 87 patients underwent EHBD resection. We used a propensity score matching analysis to reduce the selection bias inherent in retrospective observational studies. Our results showed that the median overall survival time of patients who underwent EHBD resection was longer than that of patients who did not undergo EHBD resection (25 months vs 11 months, $\mathrm{p}=0.032$ ). In addition, the median number of lymph nodes dissected in patients who underwent EHBD resection was greater than that in patients who did not undergo EHBD resection (6 vs 4, p<0.001). Furthermore, our results demonstrated that EHBD resection was an independent prognostic factor for overall survival in advanced GBC patients. Patients who underwent EHBD resection were more likely to undergo simultaneous lymph node dissection. The percentage of patients who had a least one lymph node sampled was higher in the group that underwent EHBD resection than in the group that did not (94.5\% vs 81.5\%, $\mathrm{p}<0.001) .{ }^{6}$ Moreover, EHBD resection may improve survival in patients with perineural invasion, even in the absence of biliary infiltration, according to a report by Sakamoto et al. ${ }^{10}$

At present, most of the existing studies do not support routine EHBD resection. However, routine EHBD resection may increase the number of lymph nodes dissected and allow the complete dissection of potentially invasive tissue. It is useful for TNM staging and determining the next step in treatment for patients with GBC. EHBD resection and biliary reconstruction have no severe complications; therefore, we recommend that experienced surgeons routinely perform EHBD resection in patients with advanced GBC to ensure patient safety. It remains controversial whether routine EHBD resection can improve survival. Further evaluation in randomized clinical trials and multicenter studies is needed. Internationally recognized guidelines are needed for clinical surgery programs.

\section{CONFLICTS OF INTEREST}

No potential conflict of interest relevant to this article was reported.

\section{ACKNOWLEDGEMENTS}

This work was supported by Sichuan Science and Technology Program (2018JY0019), the Fundamental Research Funds for the Central Universities (2019SCUH) and Wu Jieping Medical Foundation for Clinical Research (320.2710.1825).

\section{ORCID}

Fei Liu

https://orcid.org/0000-0002-2174-5701

$\mathrm{Fu}-\mathrm{Yu} \mathrm{Li}$ https://orcid.org/0000-0003-2755-2887

\section{REFERENCES}

1. Shimizu Y, Ohtsuka M, Ito H, et al. Should the extrahepatic bile duct be resected for locally advanced gallbladder cancer? Surgery 2004;136:1012-1017.

2. Chikamoto A, Tsuji T, Nakahara 0, et al. Cancer cells spread through lymph vessels in the submucosal layer of the common bile duct in gallbladder carcinoma. J Hepatobiliary Pancreat Surg 2009;16:557-561.

3. Kohya N, Miyazaki K. Hepatectomy of segment 4a and 5 combined with extra-hepatic bile duct resection for T2 and T3 gallbladder carcinoma. J Surg Oncol 2008;97:498-502.

4. D’Angelica M, Dalal KM, DeMatteo RP, Fong Y, Blumgart LH, Jarnagin WR. Analysis of the extent of resection for adenocarcinoma of the gallbladder. Ann Surg Oncol 2009;16:806-816.

5. Choi SB, Han HJ, Kim WB, Song TJ, Suh SO, Choi SY. Surgical strategy for T2 and T3 gallbladder cancer: is extrahepatic bile duct resection always necessary? Langenbecks Arch Surg 2013;398: 1137-1144.

6. Gani F, Buettner S, Margonis GA, et al. Assessing the impact of common bile duct resection in the surgical management of gallbladder cancer. J Surg Oncol 2016;114:176-180.

7. Araida T, Higuchi R, Hamano M, et al. Should the extrahepatic bile duct be resected or preserved in R0 radical surgery for advanced gallbladder carcinoma? Results of a Japanese Society of Biliary Surgery Survey: a multicenter study. Surg Today 2009;39:770779.

8. Yokomizo H, Yamane T, Hirata T, Hifumi M, Kawaguchi T, Fukuda $\mathrm{S}$. Surgical treatment of pT2 gallbladder carcinoma: a reevaluation of the therapeutic effect of hepatectomy and extrahepatic bile duct resection based on the long-term outcome. Ann Surg Oncol 2007; 14:1366-1373.

9. Wang JK, Ma WJ, Wu ZR, et al. Is combined extra-hepatic bileduct resection justified for advanced gallbladder carcinoma? Gastroenterol Rep (Oxf) 2019;7:426-433.

10. Sakamoto Y, Kosuge T, Shimada K, et al. Clinical significance of extrahepatic bile duct resection for advanced gallbladder cancer. $\mathrm{J}$ Surg Oncol 2006;94:298-306. 